Ann. Biol. anim. Bioch. Biophys., I970, 10 (3), 5I7-52I.

\title{
INFLUENCE DE LA NATURE DES MATIERES AZOTÉES ALIMENTAIRES SUR L'AMINO-ACIDÉMIE LIBRE DES VACHES LAITIERES
}

\author{
C. CHAMPREDON, R. PION et M. JOURNET \\ avec la collaboration technique de Madeleine Pelletier, G. Bayle et B. Marouis \\ Station d'Études des Métabolismes, \\ Station de Recherches sur l'Élevage des Ruminants, \\ Centre de Recherches de Clermont-Ferrand, 63 -Saint-Genès-Champanelle \\ Institut national de la Recherche agronomique
}

Les matières azotées alimentaires subissent sous l'action des microorganismes, un profond remaniement dans le rumen et il est difficile de prévoir les quantités des différents acides aminés et en particulier des acides aminés indispensables que l'organisme aura à sa disposition à la suite de l'ingestion d'une ration déterminée. Or, les acides aminés libres du sang sont la principale forme de transport des composés azotés dans l'organisme, et leurs concentrations dans ce tissu dépendent à chaque instant de la différence entre les apports (endogènes et exogènes), et les diverses utilisations. Un certain nombre de travaux effectués sur les ruminants ont mis en évidence le fait que les concentrations plasmatiques (Virtanen, 1969) ou sanguine (Champredon, Pion, Fauconneau, I970) des divers acides aminés libres pouvaient varier de manière sensible avec le régime. Dans la présente étude, réalisée sur des vaches laitières, nous avons déterminé l'aminoacidémie libre des animaux qui recevaient de l'ensilage de maïs et un aliment concentré apportant un complément d'azote sous forme d'urée ou de tourteau d'arachide. Les résultats ont été comparés avec ceux obtenus avec les mêmes animaux consommant uniquement de l'herbe.

\section{MÉTHODE EXPÉRIMEN'TALE}

De l'ensilage de maîs était distribué à volonté deux fois par jour du deuxième au cinquième mois de lactation à deux lots de neuf vaches de race Frisonne (JoURNET, I970). Un aliment concentré composé de luzerne déshydratée, d'orge et de minéraux, apportait un complément d'azote sous forme d'urée ou de tourteau d'arachide selon le lot, de telle façon que les rations soient isoénergétiques et isoazotées (tabl. I).

A la fin de l'expérience, les régimes expérimentaux étaient remplacés, après une période de transition de sept jours, par une distribution d'herbe. Les échantillons de sang ont été prélevés à la seringue dans la veine jugulaire sur six vaches de chaque lot alors qu'elles recevaient les régimes expérimentaux depuis cinq semaines et que les concentrés contenant de l'arachide et de l'urée étaient distribués respectivement 2 et 4 fois par jour (Champredon, Pion, Fauconneau, I969), puis sur les mêmes animaux après trois sepnaines de consommation d'herbe. Les échan- 
tillons de sang étaient mélangés immédiatement après le prélèvement à 7 volumes d'éthanol $95^{\circ}$ froid. Les acides aminés libres étaient extraits par l'éthanol $82^{\circ}$ selon une technique précédemment décrite (PAwLAK et PION, I968) et dosés par chromatographie sur colonne échangeuse d'ions au moyen d'un analyseur automatique (SPackman, Stein, Moore, 1958). L'urée sanguine était dosée par une méthode colorimétrique à la diacétyl monoxine mise au point au laboratoire (Michel, I970).

TABLEAU I

Régimes expérimentaux

(en $\mathrm{kg}$ de matière sèche ingérée par jour — moyenne par vache sur I2 semaines)

\begin{tabular}{|c|c|c|}
\hline & Lot "Urée" & Lot "Tourteau" \\
\hline Matière sèche totale $\ldots \ldots \ldots \ldots \ldots \ldots \ldots \ldots$ & 12,8 & 13,\}^{\prime}$ \\
\hline Ensilage de maïs à 25 p. 100 de M. S. . . . . . & 10,4 & 10,1 \\
\hline 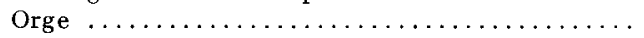 & 1,56 & 1,04 \\
\hline Luzerne déshydratée $\ldots \ldots \ldots \ldots \ldots \ldots \ldots \ldots$ & 0,70 & 1,00 \\
\hline Tourteau d'arachide $\ldots \ldots \ldots \ldots \ldots \ldots \ldots \ldots$ & 一 & 1,22 \\
\hline Urée à 42 p. 100 de $\mathrm{N} \ldots \ldots \ldots \ldots \ldots \ldots$ & 0,18 & - \\
\hline $\mathrm{N}$ Uréique en $\mathrm{p} .100 \mathrm{du} \mathrm{N}$ total $\ldots \ldots \ldots \ldots \ldots$ & 26,0 & - \\
\hline Matières azotées totales $(\mathrm{N} \times 6,25) \ldots \ldots \ldots \ldots$ & 1,83 & 1,96 \\
\hline
\end{tabular}

\section{RÉSULTATS}

La plupart des acides aminés " non indispensables ": acide aspartique, sérine plus glutamine, glycine et à un moindre degré proline et citrulline sont en concentrations plus faibles dans le sang des vaches recevant le tourteau que dans celui des animaux consommant l'urée, alors que les teneurs en alanine et ornithine sont très voisines dans les deux cas ; seul l'acide glutamique se trouve en concentration plus élevée chez les vaches consommant le tourteau.

Parmi les acides aminés "indispensables " et " semi-indispensables " seule l'arginine est en concentration nettement plus élevée chez les animaux recevant l'urée. La thréonine, la méthionine et la tyrosine $\mathrm{y}$ sont en concentrations très légèrement supérieures à celles que l'on observe dans l'autre lot, alors que les teneurs en valine, en isoleucine, en leucine et moins nettement en phénylalanine et en histidine sont plus importantes dans le cas du lot " tourteau n. Il n'y a pas de différence entre les deux groupes en ce qui concerne la lysine.

Ces résultats sont en accord avec ceux qui avaient été obtenus précédemment avec 6 vaches laitières recevant depuis I 2 semaines les mêmes régimes expérimentaux (ChAmpredon, PIoN, Fauconneau, I969) pour certains acides aminés tels que la thréonine et l'histidine. Ils en diffèrent notamment pour la valine, l'isoleucine, l'ornithine, la lysine et l'arginine dont les concentrations sanguines étaient plus élevées.

Les acides aminés libres sont généralement en concentrations sanguines plus élevées lorsque les animaux reçoivent de l'herbe que lorsqu'ils consomment les deux régimes expérimentaux. Ce sont l'alanine, la valine, l'isoleucine la leucine, la tyrosine, l'ornithine, la lysine et l'histidine dont les teneurs augmentent le plus dans le sang des animaux des deux lots à la suite du changement de régime. Il faut y ajouter la thréonine et la phénylalanine chez les animaux ayant reçu l'urée, et l'acide aspartique ainsi que la méthionine pour l'autre lot. Quelques acides aminés ont cependant des teneurs plus basses avec l'herbe qu'avec les régimes étudiés. Il s'agit, pour les deux groupes d'animaux, d'acides aminés non indispensables : sérine plus glutamine, citrulline chez les animaux ayant reçu le tourteau, acide aspartique et glycine pour l'autre lot. L'arginine reste à 
un taux relativement bas dans les deux cas. De plus, à l'exception de la thréonine et surtout de l'ornithine, les teneurs en acides aminés libres du sang des deux groupes sont très voisines après trois semaines de consommation d'herbe.

Les teneurs en urée sanguine des différents prélèvements sont voisines et ne sont jamais très élevées.

\section{TABLEAU 2}

Aminoacidémie libre des vaches laitières

(mg/Ioog de sang frais)

\begin{tabular}{|c|c|c|c|c|}
\hline & \multicolumn{2}{|c|}{ Régime "Urée" } & \multicolumn{2}{|c|}{ Régime "Tourteau" } \\
\hline & Rég. Expér. & Herbe & Rég. Expér. & Herbe \\
\hline Ac. aspartique $\ldots \ldots \ldots \ldots \ldots$ & 1,00 & 0,95 & $0,3 \tilde{3}$ & 0,61 \\
\hline Thréonine................ & 0,96 & 1,28 & 0,85 & 0,99 \\
\hline Sérine + Glutamine $\ldots \ldots \ldots$ & $4, \geq 7$ & 3,01 & 3,41 & 3,07 \\
\hline Ac. giutamique $\ldots \ldots \ldots \ldots \ldots$ & 1,28 & & 1,73 & 1,73 \\
\hline Proline $\ldots \ldots \ldots \ldots \ldots \ldots \ldots$ & 1,03 & & 0,92 & 0,99 \\
\hline Citrulline $\ldots \ldots \ldots \ldots \ldots \ldots$ & 0,93 & & 0,85 & 0,61 \\
\hline Glycine $\ldots \ldots \ldots \ldots \ldots \ldots$ & 2,76 & 2,42 & 2,43 & 2,56 \\
\hline Alanine $\ldots \ldots \ldots \ldots \ldots \ldots$ & 1,49 & 2,12 & 1,52 & 2,14 \\
\hline Valine $\ldots \ldots \ldots \ldots \ldots \ldots \ldots$ & 1,34 & 2,66 & 1,60 & 2,65 \\
\hline Méthionine $\ldots \ldots \ldots \ldots \ldots \ldots$ & 0,42 & 0,44 & 0,35 & 0,51 \\
\hline Isoleucine $\ldots \ldots \ldots \ldots \ldots \ldots$ & 0,77 & 1,54 & 1,00 & 1,47 \\
\hline Leucine .................. & 1,10 & 1,70 & 1,24 & 1,63 \\
\hline Tyrosine $\ldots \ldots \ldots \ldots \ldots \ldots$ & 0,88 & 1,18 & 0,81 & 0,98 \\
\hline Phénylalanine $\ldots \ldots \ldots \ldots \ldots \ldots$ & 0,62 & 0,89 & 0,74 & 0,86 \\
\hline Ornithine $\ldots \ldots \ldots \ldots \ldots \ldots$ & 0,37 & 0,48 & 0,39 & 0,91 \\
\hline Lysine . . . . . . . . . . . & 0,81 & 1,19 & 0,82 & 1,13 \\
\hline Histidine $\ldots \ldots \ldots \ldots \ldots \ldots$ & 0,68 & 0,92 & 0,78 & 0,98 \\
\hline Arginine $\ldots \ldots \ldots \ldots \ldots \ldots$ & 0,48 & 0,48 & 0,36 & $0, \mathbf{4}^{\prime} \mathbf{t}$ \\
\hline Urée $\ldots \ldots \ldots \ldots \ldots \ldots \ldots$ & 36 & 37 & 35 & 34 \\
\hline
\end{tabular}

\section{DISCUSSION}

Les concentrations des acides aminés "indispensables "dans le sang des animaux des deux lots étaient assez peu différentes, ce qui ne signifie pas que les apports d'acides aminés par leur tractus digestif étaient les mêmes dans les deux cas. En effet, les quantités ingérées par les animaux consommant l'urée étaient plus faibles au début de l'expérience, ce qui a limité en partie eur production laitière à un niveau inférieur de plus de ıo p. Ioo à celle des autres vaches, et a entrainé une baisse de la teneur en matières azotées de leur lait; les besoins en acides aminés correspondant à la synthèse des protéines du lait et par conséquent les quantités prélevées par la glande mammaire étaient donc plus faibles chez les animaux du premier lot.

Les quantités d'acides aminés fournis par leur tube digestif sont donc probablement plus faibles et il doit y avoir chez les animaux un certain gaspillage d'azote qui est peut-être en relation avec la cinétique de l'ammoniogenèse dans le rumen; une libération rapide d'ammoniac, supérieure aux possibilités de synthèse protéique des bactéries, entraîne un passage dans le sang d'ammoniac qui ne reviendra que partiellement dans le rumen sous forme d'urée, d'où une production plus faible d'acides aminés. L'étude cinétique effectuée précédemment, a montré qu'il 
y avait donc une certaine discontinuité des apports d'acides aminés dans le sang par le tractus digestif.

Les concentrations en certains acides aminés "indispensables" tels que la valine, l'isoleucine, la lysine et l'arginine, sont plus faibles que celles qui avaient été observées précédemment avec d'autres vaches consommant les mêmes régimes expérimentaux depuis I 2 semaines au lieu de 5. Cette différence peut avoir plusieurs causes; les animaux avaient une production laitière plus faible après $I 2$ semaines qu'après 5 semaines d'expérience, et par conséquent les proportions d'azote distribuées sous forme d'urée ou de tourteau d'arachide étaient alors moins importantes. De plus, ils consommaient les régimes expérimentaux depuis plus longtemps, et pouvaient avoir une flore mieux adaptée à leur utilisation et plus apte à satisfaire leurs besoins en acides aminés, et cela d'autant plus que les apports énergétiques étaient alors légèrement excédentaires.

Les différences de teneurs en acides aminés libres liées à la nature du complément azoté sont dans l'ensemble en accord avec les données de la littérature. Virtanen (i969) a constaté chez les vaches laitières que l'utilisation d'urée comme seul apport azoté se traduisait par des teneurs plasmatiques en de nombreux acides aminés " indispensables " tels que la valine, l'isoleucine, la leucine, la lysine, et surtout l'histidine plus faibles que dans le cas d'une alimentation normale. Les différences que nous avons observées portent généralement sur les mêmes acides aminés, mais la faiblesse des teneurs liées à l'ingestion d'urée est naturellement moins accusée que celle qui avait été mise en évidence par cet auteur puisque l'azote uréique ne représentait que $29 \mathrm{p}$. 100 de l'azote total ingéré par nos animaux, au moment du prélèvement. D'autres auteurs (OLTJEN et Putnam, 1966) ont montré que les différences entre les teneurs en acides aminés libres du plasma de bovins en croissance recevant de l'urée ou du tourteau de soja portaient principalement sur les acides aminés à chaîne ramifiée et sur la phénylalanine. Le fait que nous observions des différences pour un plus grand nombre d'acides aminés avec toutefois des écarts relativement peu importants peut être dû à la nature des tourteaux utilisés.

Les augmentations des teneurs en acides aminés "indispensables » observées lors du passage à l'herbe traduisent un certain excédent des apports en acides aminés par rapport aux besoins, ce qui peut être dû en partie à l'ingestion d'un régime plus favorable aux synthèses des corps microbiens, et au fait que les quantités d'azote ingéré étaient supérieures aux besoins des animaux compte tenu de leur production laitière. Les augmentations de teneurs en acides aminés libres du sang des animaux liées à la modification de leurs régimes alimentaires sont généralement similaires à celles que nous avions observées chez la Chèvre en lactation (CHAMpredon, Pion, FaUCONNEAU, 1970); chez des animaux à besoins élevés, les concentrations en acides aminés du sang augmentaient de manière importante lorsqu'une ration composée de concentré et d'herbe était substituée à un régime constitué de foin et du même concentré. Toutefois, les teneurs en lysine et en histidine ne se trouvaient pas augmentées ou étaient même diminuées, ce qui peut être dû au fait que les chèvres étudiées étaient en pleine production laitière et avaient des besoins azotés proportionnellement plus élevés que ceux des vaches de la présente expérience.

Reçu pour publication en avril 1970.

\section{REMERCIEMENTS}

Nous remercions M. M.-C. Michel qui a bien voulu effectuer les dosages d'urée sanguine. 


\section{SUMMARY \\ EFFEC'T OF THE ORIGIN OF DIETARY NITROGEN ON FREE \\ AMINOACID BLOOD RATE IN THE COW}

Two groups of 9 lactating cows were given a corn silage diet added with a concentrate of barley, alfafa meal, minerals, and either urea or peanut meal. At the end of the experimental period, all animals were given orchard grass for three weeks. In order to determine free-aminoacid rates, blood samples were taken from the jugular veins of 6 animals from each group at week 5 of the experimental diet and week 3 of the grass diet.

The rates of most essential aminoacids were lower in the urea group blood than in the peanut meal one. There was a consistent increase in the essential free-aminoacid blood rates after the three-week grass diet.

\section{RÉFÉRENCES BIBLIOGRAPHIQUES}

Champredon C., Pion R., Fauconneau G., i969. L'aminoacidémie libre des bovins. Variations au cours de la journée. C. R. Acad. Sci., Paris Série D, 269, 2029-2032.

Champredon C., Pion R., Fauconneau G., ig7o. L'aminoacidémie libre des chèvres. Variations avec le régime. C. R. Soc. Biol. (sous presse).

Journet M. (Én préparation).

Michel M.-C. (En préparation).

Oltjen R. R., Putnam P. A., I966. Plasma amino acids and nitrogen retention by steers fed purified diets containing urea or isolated soy protein. J. Nutr., 89, 385-39r.

PAwl.AK M., Pion R., I 968 . Influence de la supplémentation des protéines du blé par des doses croissantes de lysine sur la teneur en acides aminés libres du sang et du muscle du rat en croissance. Ann. Biol, anim. Bioch. Biophys., 8, 517-530.

Spackman D. H., Stein W. H., Moore J., I958. Automatic recording apparatus for use in the chromatography of amino acids. Analyt. Chem., 30, I $190-\mathbf{1} 206$.

Virtanen A. I., I 969 . On nitrogen metabolism in milking cows. Fedn. Proc., 28, 232-240. 